\title{
Heart Rate Variability and Pulse Pressure Amplification: Lessons from Diabetic Patients
}

\author{
Agne Laucyte-Cibulskiene \\ Centre of Nephrology, Vilnius University Hospital Santaros Clinics, and Clinic of \\ Gastroenterology, Nephrourology and Surgery, Faculty of Medicine, Vilnius University, \\ Vilnius, Lithuania
}

The study by Di Daniele et al. "Lower Heart Rate Variability Is Associated with Lower Pulse Pressure Amplification: Role of Obesity" [1] indirectly gives us insight into heart rate variability (HRV) and pulse pressure amplification (PPA) in mainly hypertensive diabetic patients in the context of obesity. HRV is one of the first symptoms of cardiac autonomic neuropathy in diabetes, which is also associated with blood pressure dysregulation and left ventricular hypertrophy [2]. Furthermore, recent studies have also shown increased PPA in type 2 diabetic hypertensive patients [3], suggesting vascular remodeling due to increased arterial stiffness as another possible etiology of HRV. It would be interesting to analyze the influence of obesity on HRV and PPA in diabetic patients. The authors of the article reveal that obesity is significantly associated with HRV in models adjusted for diabetes mellitus and other confounding variables. Unfortunately, they do not elaborate on these results.

The authors discuss the inaccuracy of body mass index for defining obesity. But there are more issues regarding this, for example the role of fat-free mass (FFM), fat mass (FM), and the autonomic nervous system [4] in obese individuals, especially when bearing in mind the above-mentioned mainly diabetic study population. There is a debate about the superiority of bioelectrical impedance analysis over dual X-ray absorptiometry, but both methodologies have errors in measuring FFM and FM in obese patients. Although there is evidence that body shape index [5] could better identify sarcopenic obesity than body mass index or waist circumference, this relationship was observed only in men. Further studies looking for a link between HRV and FFM and FM in obese and lean individuals are encouraged.

\section{Disclosure Statement}

I have no actual or potential conflict of interests in relation to this publication. 
Laucyte-Cibulskiene et al.: Heart Rate Variability and Pulse Pressure Amplification: Lessons from Diabetic Patients

\section{References}

1 Di Daniele N, Tesauro M, Mascali A, Rovella V, Scuteri A: Lower heart rate variability is associated with lower pulse pressure amplification: role of obesity. Pulse 2017, DOI: $10.1159 / 000479701$.

2 Pop-Busui R: Cardiac autonomic neuropathy in diabetes. Diabetes Care 2010;33:434-441.

-3 Yannoutsos A, Ahouah M, Tubiana CD, Topouchian J, Touboul C, Safar ME, et al: Hemodynamic parameters in hypertensive diabetic patients. J Hypertens 2016;34:1123-1131.

-4 Weise CM, Thiyyagura P, Reiman EM, Chen K, Krakoff J: Fat-free body mass but not fat mass is associated with reduced gray matter volume of cortical brain regions implicated in autonomic and homeostatic regulation. NeuroImage 2013;64:712-721.

-5 Dhana K, Koolhas C, Schoufour J, Rivadeneira F, Hofman A, Kavousi M, et al: Association of anthropometric measures with fat and fat-free mass in the elderly: the Rotterdam study. Maturitas 2016;88:96-100. 